\title{
ADESÃO À TERAPIA NUTRICIONAL POR PACIENTES DIABÉTICOS INTERNADOS EM UM HOSPITAL PÚBLICO DO MUNICÍPIO DE CURITIBA-PR
}

\author{
ADHERENCE TO NUTRITIONAL THERAPY OF DIABETIC PATIENTS \\ HOSPITALIZED IN A PUBLIC HOSPITAL IN THE CITY OF CURITIBA, PARANÁ
}

\section{Nayana Cavassim do Nascimento ${ }^{{ }^{*}}$, Marília Rizzon Zaparolli2 ${ }^{*}$, Deise Regina Baptista ${ }^{3 *}$, Stela Adami Vayego ${ }^{*}$}

'nayana.cavassim@gmail.com, ${ }^{2}$ mahzaparolli@gmail.com, ${ }^{3}$ deiseregina@ufpr.br, ${ }^{4}$ vayego@ufpr.br *Universidade Federal do Paraná - Curitiba (PR), Brasil.

Data de entrada do artigo: 23/02/2013 Data de aceite do artigo: 19/03/2014

\section{RESUMO}

Introduçáo: $\mathrm{O}$ diabetes é uma doença crônica de alta prevalência que pode desencadear diversas complicaçôes. Objetivo: Verificar a adesão à terapia nutricional (TN) como forma de tratamento para pacientes diabéticos internados no Hospital de Clínicas (HC)/UFPR e avaliar o estado nutricional (EN) dos indivíduos. Materiais e Métodos: Estudo transversal realizado com pacientes diabéticos internados nas clínicas médicas masculina e feminina do HC/UFPR com idade superior a 18 anos. Os dados foram coletados por meio da aplicação de um questionário, o qual apresentava informaçóes como peso e altura dos pacientes e indagava a respeito das formas de tratamento utilizadas na comorbidade, destacando a TN. Os dados foram planilhados no programa Microsoft Excel ${ }^{\circ} \mathrm{e}$ analisados utilizando o programa Bioestat ${ }^{\circ}$. Para verificar a associação entre variáveis aplicou-se o teste $\mathrm{G}$ de Williams ao nível 5\% de significância. Resultados: A amostra foi composta por 34 pacientes, a maioria do sexo feminino $(55,88 \%)$, diabéticos tipo $2(88,2 \%)$, com diagnóstico nutricional de obesidade ( $41,18 \%$ em > de 60 anos) e sobrepeso $(64,7 \%$ em < de 60 anos). Ao analisar as variáveis TN e EN, observa-se que não houve associação estatística significativa $(p>0,05)$, resultando em $p=0,6250$. Entre os pacientes, 58,8\% afirmou realizar TN, sendo que apenas $20 \%$ possuía prescrição dietética de nutricionista. Conclusão: A maior parte da amostra foi composta por pacientes com excesso de peso e que afirmaram realizar TN, porém observou-se baixa prevalência da prescrição dietética. Os resultados evidenciam a importância da inserção do nutricionista no acompanhamento de pacientes diabéticos para garantir a manutenção e melhora do estado nutricional. Novos estudos que abordem o assunto devem ser realizados.

Palavras-chave: terapia nutricional; estado nutricional; diabetes mellitus.

\section{ABSTRACT}

Introduction: Diabetes is a chronic disease of high prevalence that can cause several complications. Objective: To assess the adherence to nutritional therapy (TN) as a treatment approach for diabetic patients hospitalized at the Hospital das Clínicas (HC)/UFPR and assess the nutritional status (EN) of the subjects. Materials and Methods: Cross-sectional study was carried out with diabetic patients hospitalized in male and female medical clinics at HC/UFPR aged over 18. Data were collected through a questionnaire, which presented information such as weight and height of the patients and inquired about the treatment used in comorbidity, emphasizing TN. Data were tabulated on Microsoft Excel ${ }^{\circ}$ and analyzed using Bioestat ${ }^{\circ}$. To determine the association between variables the Williams' $G$ test was applied at a 5\% significance level. Results: The sample consisted of 34 patients, the majority were female (55.88\%), type 2 diabetics, with nutritional diagnosis of obesity ( $41.18 \%$ in $>60$ years old) and overweight (64.7\% in $<60$ years old). By analyzing TN and EN variables, no statistically significant association was observed ( $p>0,05$ ), resulting in $p=0,6250$. Among the patients, $58.8 \%$ said to carry out $T N$, being that 
only $20 \%$ had a dietary prescription from a nutritionist. Conclusions: The majority of the sample was composed of patients with overweight and who reported receiving, but a low prevalence of dietary prescription was observed. The results show the importance of inserting a nutritionist in the monitoring of diabetic patients to ensure the maintenance and improvement of nutritional status. Further studies that address the subject should be conducted.

Keywords: nutrition therapy; nutritional status; diabetes mellitus.

\section{Introdução}

O diabetes mellitus (DM) é uma doença metabólica caracterizada por hiperglicemia resultante de defeitos na secreção ou na ação do hormônio insulina, sendo que em alguns casos tanto a ação quanto a secreção estão comprometidas ${ }^{1,2}$. A insulina é um hormônio indispensável ao organismo, pois regula os níveis glicêmicos. Se este hormônio estiver em falta no organismo, a glicose irá se acumular no plasma fazendo que algumas complicaçóes como enfermidades cardíacas, feridas que não cicatrizam e problemas de visão possam surgir ${ }^{3}$.

Devido à alta prevalência, assim como o fato de desencadear complicaçôes crônicas que afetam a qualidade de vida do portador e geram alto custo para os sistemas de saúde, o DM é considerado um problema de saúde mundial ${ }^{4}$. A doença tem despertado grande atençáo nos últimos anos por causa de alguns fatores que têm aumentado sua proporção na população, como o maior consumo de alimentos hipercalóricos, o acelerado processo de urbanizaçáo, o sedentarismo, a mudança no estilo de vida e a obesidade ${ }^{5}$.

Em 1542, foi realizado o primeiro trabalho que abordava a terapia nutricional no tratamento do DM. Essa dieta baseava-se na exclusão de alimentos ricos em carboidratos, porém não foi bem tolerada pelo fato de possuir grande quantidade de gordura. Em 1674, Thomas Willis começou a introduzir carboidratos na terapia nutricional do DM com o objetivo de compensar as perdas de glicose na urina, porém manteve baixas as recomendaçóes de mono e dissacarídeos ${ }^{6}$. Atualmente, a Sociedade Brasileira de Diabetes, em sua Diretriz para o Acompanhamento e Tratamento do Diabetes Mellitus, considera que a mudança no estilo de vida e a orientação nutricional são terapias de primeira escolha. As recomendaçóes do plano alimentar incluem o fracionamento correto das refeiçóes e o aumento no consumo de alimentos naturais, como vegetais, frutas e cereais integrais, que também contribuem para o aumento no consumo de fibras?.

No tratamento do diabetes, a terapia nutricional tem suma importância associada à prática de atividades físicas e medicação correta. O papel do nutricionista como profissional de saúde é orientar o paciente e elaborar um plano alimentar de acordo com suas necessidades nutricionais, hábitos alimentares e situação socioeconômica ${ }^{4}$.
Por meio de um plano alimentar, o portador de DM obtém diversos benefícios. Além de ajudar a manter os níveis de glicose normalizados, a alimentação correta auxilia na redução do risco de desenvolver complicaçôes vasculares por permitir o controle nos níveis de lipídeos e lipoproteínas.

A adesão ao tratamento é definida como uma mudança comportamental em busca de hábitos de vida saudáveis, e o DM está entre as doenças com menores taxas de adesáo ao tratamento por se tratar de uma doença crônica e exigir diversos cuidados ${ }^{9}$.A baixa adesão ao tratamento por portadores de doenças crônicas é um dos problemas mais relatados por profissionais de saúde. Isso é observado devido ao fato de os tratamentos serem a longo prazo e exigirem mudanças no estilo de vida do paciente ${ }^{10}$. O padrão alimentar muitas vezes é difícil de ser modificado por estar envolvido com questôes emocionais e históricas ${ }^{11}$. É importante que a conscientização a respeito da adesão ao tratamento dietético seja realizada pelos profissionais de saúde, porém deve haver um cuidado a respeito das crenças e costumes que os pacientes possuem ${ }^{12}$.

Assim como para o restante da população, o objetivo da dieta para portadores de diabetes é atingir o estado nutricional ideal ${ }^{13}$. Os portadores de diabetes devem adequar ao seu dia a dia o tratamento que lhes foi prescrito e que muitas vezes não é aderido pelo fato de os indivíduos serem os principais responsáveis nessa mudança de hábitos comportamentais e alimentares que devem ser incorporados no cotidiano ${ }^{1}$.

O objetivo deste trabalho foi verificar a adesão à terapia nutricional (TN) como forma de tratamento em pacientes diabéticos internados no Hospital de Clínicas (HC)/UFPR e avaliar o estado nutricional (EN) dos indivíduos.

\section{Materiais e Métodos}

O presente estudo possui um delineamento transversal e foi realizado com pacientes diabéticos internados no HC/UFPR. A coleta de dados foi feita por meio da aplicação de um questionário contendo informações sobre dados pessoais, avaliação antropométrica (peso, altura, Índice de Massa Corpórea e dobras cutâneas), prática de atividade física, uso de insulina e 
antidiabéticos orais e realização de terapia nutricional prévia ao internamento.

Os questionamentos a respeito da terapia nutricional foram feitos de forma subjetiva, por meio de perguntas direcionadas aos próprios pacientes que abordavam o seguimento ou não de um planejamento dietético e quem havia realizado a prescrição. Para os pacientes que afirmavam possuir prescrição dietética de profissional de saúde, eram realizados outros questionamentos a respeito do seguimento das recomendações e dificuldades encontradas. Os dados da avaliação antropométrica foram obtidos através dos prontuários dos pacientes, uma vez que a avaliação nutricional já faz parte da rotina estabelecida pelas nutricionistas do referido hospital.

Antes da aplicação do questionário, o objetivo do estudo era explicado, e a coleta de dados só era realizada se o paciente concordasse em participar da pesquisa por meio da assinatura do Termo de Consentimento Livre e Esclarecido (TCLE). Este projeto foi aprovado pelo Comitê de Ética em Pesquisa com Seres Humanos do HC/UFPR, registro no 2332.226/2010-09.

Pelo fato de a pesquisa ter sido realizada nas clinicas médicas masculina e feminina, o publico alvo consistiu somente em pacientes maiores de 18 anos.

A coleta de dados foi realizada no período de novembro de 2011 a março de 2012 no período matutino e vespertino, sendo a duração da aplicação do questionário de aproximadamente 15 minutos e o preenchimento dos dados realizado pelas próprias pesquisadoras.

O diagnóstico nutricional dos pacientes foi obtido pelo Índice de Massa Corpórea com os pontos de corte propostos pela Organização Mundial de Saúde ${ }^{14}$ para indivíduos entre 18 e 60 anos, e para indivíduos maiores de 60 anos foram utilizados os pontos de corte propostos pelo Nutrition Screening Initiative e $^{15}$ recomendados pelo Ministério da Saúde. Os dados foram digitados em planilhas eletrônicas por meio do programa Microsoft $\operatorname{Excel}^{\circledR} \mathrm{e}$ analisados utilizando o programa Bioestat ${ }^{\circ}$. A análise estatística dos dados foi realizada determinando as frequências e porcentagens das diversas variáveis categóricas em estudo. A existência de associação entre variáveis de interesse foi verificada a partir de tabelas de contingência mediante o teste G de Williams ao nível de significância de 5\%.

\section{Resultados}

A amostra foi composta por 34 pacientes, sendo a maioria do sexo feminino $(55,9 \%)$. O diabetes tipo 2 foi encontrado em maior frequência $(88,2 \%)$, sendo que somente $11,8 \%$ dos pacientes eram portadores do diabetes tipo 1.
De acordo com a classificação do estado nutricional, conforme ilustrado na Tabela 1 , dos pacientes com idade inferior a 60 anos, a maior prevalência foi encontrada para obesidade, correspondendo a $41,18 \%$ do total de indivíduos nessa faixa etária. Dos 17 indivíduos com idade superior a 60 anos, a maior frequência foi encontrada para o sobrepeso, correspondendo a $64,7 \%$ dos pacientes.

Tabela 1: Classificação do estado nutricional de acordo com o IMC para adultos e idosos

\begin{tabular}{lccccc}
$\begin{array}{l}\text { Menores de } \\
\mathbf{6 0} \text { anos }\end{array}$ & $\mathbf{N}$ & $\mathbf{\%}$ & $\begin{array}{c}\text { Maiores de } \\
\mathbf{6 0} \text { anos }\end{array}$ & $\mathbf{N}$ & $\mathbf{\%}$ \\
\hline Desnutrição & 3 & 17,65 & Baixo peso & 3 & 17,65 \\
Eutrofia & 3 & 17,65 & Eutrofia & 3 & 17,65 \\
Sobrepeso & 4 & 23,52 & Sobrepeso & 11 & 64,70 \\
Obesidade & 7 & 41,18 & & & \\
Total & 17 & 100 & & 17 & 100 \\
\hline
\end{tabular}

Ao serem questionados a respeito da forma de tratamento que realizavam para controlar o diabetes, os seguintes resultados foram obtidos, conforme ilustra a Tabela 2.

Tabela 2: Formas de tratamento utilizadas no controle glicêmico pelos pacientes diabéticos

\begin{tabular}{ccc}
\hline Faz dieta & Frequência & $\%$ \\
\hline Sim & 20 & 58,8 \\
Não & 14 & 41,2 \\
\hline Pratica atividade física & & \\
\hline Sim & 11 & 32,4 \\
Não & 23 & 67,7 \\
\hline Uso de antidiabéticos & & \\
orais & & 73,6 \\
Sim & 25 & 26,5 \\
\hline Não & 9 & \\
\hline Uso de insulina & & 47,1 \\
Sim & 16 & 52,9 \\
Não & 18 &
\end{tabular}

Dentre os 34 pacientes entrevistados, 58,8\% afirmaram realizar terapia nutricional como parte do tratamento, e 41,2\% relataram não realizar nenhum tipo de controle alimentar. Com relação à prática de atividade física, foi encontrada a maior frequência para aqueles que não realizavam exercícios físicos, correspondendo a $67,7 \%$ do total de entrevistados.

Quanto à terapia medicamentosa, pode-se notar que a prevalência foi de pacientes que faziam uso de 
antidiabéticos orais $(73,5 \%)$, e o uso de insulina foi relatado por $47,1 \%$ dos entrevistados.

Os pacientes que afirmaram realizar terapia nutricional para controle da glicemia foram questionados a respeito de quem havia prescrito a dieta seguida. Diante deste propósito, foram obtidos os resultados mostrados na Tabela 3.

Tabela 3: Prescrição dietética dos indivíduos que realizavam terapia nutricional como forma de tratamento

\begin{tabular}{ccc}
\hline Quem prescreveu a dieta & N & $\%$ \\
\hline Sem prescrição & 10 & 50 \\
(mídia e cultura popular) & & \\
Médico & 6 & 30 \\
Nutricionista & 4 & 20 \\
TOTAL & 20 & 100 \\
\hline
\end{tabular}

Metade da população pesquisada que afirmou realizar dieta não possuía prescrição dietética de profissional da saúde, sendo que os próprios estabeleciam um cardápio com base na mídia e na cultura popular. Vale ressaltar que dos $50 \%$ que afirmaram aderir à dieta como forma de tratamento, somente $20 \%$ teve prescriçáo realizada por nutricionista.

Tabela 4: Recomendações realizadas por profissionais de saúde e taxa de seguimento pelos pacientes diabéticos

\begin{tabular}{lcc}
\hline Segue as recomendaçóes: & N & $\%$ \\
\hline Sim & 5 & 50 \\
Parcialmente & 5 & 50 \\
TOTAL & 10 & 100 \\
\hline Recomendaçóes que náo são & & \\
seguidas & 2 & 40 \\
\hline Diminuir o consumo de doces & 2 & 40 \\
Diminuir o consumo de massas & 1 & 20 \\
Preferir alimentos integrais & 5 & 100 \\
TOTAL & & \\
\hline Presença de modificaçóes positivas & & \\
após ter iniciado a dieta & 9 & 90 \\
\hline Sim & 1 & 10 \\
Não & 10 & 100 \\
TOTAL & & \\
\hline Indicaria a terapia nutricional para & & \\
outros pacientes & 10 & 100 \\
\hline Sim & 0 & 0 \\
Não & 10 & 100 \\
TOTAL &
\end{tabular}

De acordo com a Tabela 4, dos pacientes que possuíam prescrição dietética de profissional de saúde, 50\% realizavam corretamente a dieta, afirmando seguir todas as recomendaçóes, e 50\% responderam seguir parcialmente. As dificuldades mais prevalentes citadas pelos pacientes que não seguiam todas as recomendaçóes dietéticas foram a redução no consumo de doces e de massas, resultando ambos em $40 \%(n=2)$ do total.Dos 10 participantes que realizavam terapia nutricional com orientaçáo do profissional de saúde, $90 \%$ relataram sentir modificaçôes positivas após terem iniciado a dieta, e $100 \%$ afirmaram que indicariam a terapia nutricional para outros pacientes.

Ao serem questionados sobre o conhecimento a respeito da alimentação para portadores de diabetes, $94,1 \%$ tinham conhecimento a respeito da existência de alimentos restritos aos portadores de diabetes, sendo que somente $5,9 \%$ negaram conhecimento sobre o assunto.

Não foi encontrada associação estatística significativa entre terapia nutricional e estado nutricional $(\mathrm{p}=0,6250)$, conforme mostra a Figura 1.

Figura 1: Associação entre terapia nutricional e estado nutricional na amostra pesquisada

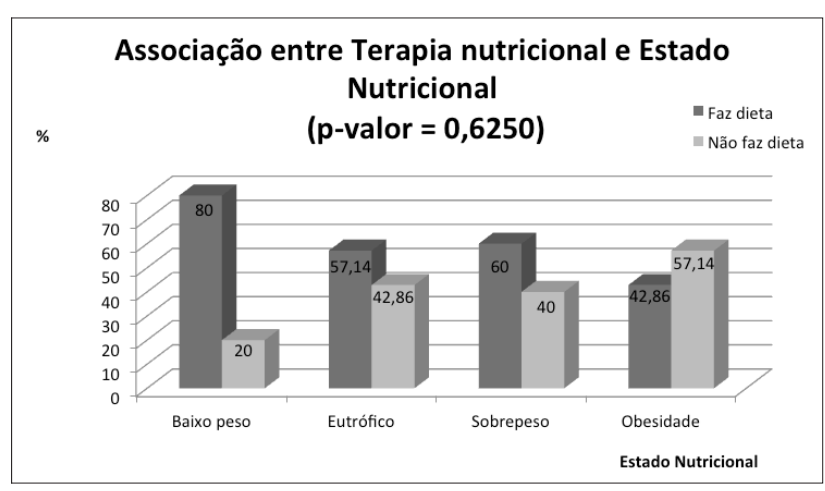

\section{Discussão}

O número de portadores de diabetes aumentou de forma significativa nos últimos anos. Isso ocorreu por diversos fatores, entre eles o aumento na expectativa de vida da populaçáo, o que favorece o aparecimento de doenças crônicas não transmissíveis, e a transição nutricional, processo que vem se desenvolvendo e está aumentando o número de obesos no mundo ${ }^{16}$.

Sabe-se que o sobrepeso e a obesidade são fatores que contribuem para o aparecimento do diabetes tipo $2^{16}$, e como obervado no presente estudo, a maior parcela da população estudada apresentava excesso de peso.

A identificação destes pacientes é fator primordial para que interversóes na área da saúde sejam feitas, reduzindo o custo necessário para o tratamento da doença e as complicações decorrentes ${ }^{17}$. Pacientes portadores 
do diabetes tipo 2 apresentam maiores chances de desenvolverem doenças cardiovasculares, fator que pode ser atribuído muitas vezes à presença de dislipidemia e hipertensão ${ }^{18}$.

Para que o tratamento no controle do diabetes seja efetivo é necessário que todas as medidas sejam tomadas, sendo que o paciente tem autonomia de seguir ou não as prescrições realizadas pelo profissional de saúde, tornando-se o agente principal no processo de tratamento ${ }^{19}$. No presente estudo, foi observado que a população em questão não realizava o tratamento de forma conjunta. Pode-se verificar que a terapia medicamentosa foi a mais citada, seguida pelo controle alimentar. A prática de atividades físicas era realizada por uma pequena parcela de pacientes, sendo que em alguns casos a justificativa da não realização se dava pela presença de outras doenças que dificultavam a realização de exercícios. Sabe-se que muitas vezes a baixa realização de terapia nutricional por pacientes diabéticos ocorre por exigir uma mudança dos hábitos alimentares que está ligada intimamente a fatores culturais e emocionais ${ }^{11}$. Vários fatores contribuem para que as pessoas acreditem que o controle alimentar é a fração mais difícil de ser seguida no tratamento do DM. Diversos planos alimentares sáo estabelecidos levando em consideraçáo somente a comorbidade e não o paciente como um todo, fazendo que as recomendações não sejam seguidas, pois aspectos pessoais importantes são descartados ${ }^{20}$. Além disso, a prática dietética pelos portadores de diabetes é vista como restritiva, sendo algo muito diferente do que costumavam ingerir ${ }^{21}$. No estudo realizado, pôde-se observar que dentre os pacientes que relataram realizar controle alimentar e possuíam prescrição dietética elaborada por profissional de saúde, $50 \%(n=5)$ disseram seguir parcialmente as recomendaçóes. Neste contexto, o paciente deve ser conscientizado sobre a importância do correto seguimento da terapêutica que lhes foi transmitida, e os profissionais de saúde devem esclarecer as dúvidas relacionadas à doença, pois isto interfere diretamente na continuidade do tratamento ${ }^{21}$.

A terapia nutricional deve ser baseada no objetivo de reduzir a morbimortalidade e aumentar a qualidade de vida dos portadores ${ }^{6}$.

Em um estudo feito por Villas-Boas ${ }^{9}$, buscou-se avaliar a adesão ao tratamento por portadores de diabetes tipo 2. A amostra foi constituída por $162 \mathrm{pa}-$ cientes com idade superior a 40 anos, frequentadores do Ambulatório de Endocrinologia e Metabologia do Hospital das Clínicas da Faculdade de Medicina de Ribeirão Preto. Assim como no presente estudo, a maior parcela da população foi composta por mulheres (58\%). $\mathrm{O}$ tratamento medicamentoso foi encontrado em grande parte da população, sendo que $64,8 \%$ realizavam a terapia associando hipoglicemiante oral e insulina. A média obtida para terapia nutricional e exercícios físicos foi de 4,34 ( $\mathrm{DP}=1,34)$, mostrando assim a baixa adesão a estas formas de tratamento.

Verificou-se no presente estudo que dentre os pacientes que afirmaram realizar controle alimentar como forma de tratamento, somente $20 \%(n=4)$ disseram ter prescrição dietética do profissional nutricionista. Vale ressaltar que o nutricionista como profissional de saúde habilitado para realizar a orientaçâo dietética é um fator essencial no tratamento do paciente diabético e deve ser parte integrante do processo educativo do portador, pois a mudança nos hábitos alimentares favorece o controle glicêmico e metabólico ${ }^{22,23}$.

\section{Conclusão}

A maior parte da amostra foi composta por pacientes que apresentavam excesso de peso, fator que pode ser associado à alta prevalência de diabéticos tipo 2 na amostra. A maioria dos pacientes afirmou realizar terapia nutricional como forma de tratamento $(58,82 \%)$, porém observouse que grande parte da amostra não possuía prescrição dietética de profissional habilitado, no caso o nutricionista. Esse fato implica na incorreta ou náo realização deste modo de tratamento, tendo em vista que a prática alimentar torna-se baseada na cultura popular. Os resultados evidenciam a importância da inserção do nutricionista no acompanhamento de pacientes diabéticos para garantir a melhora e manutenção do estado nutricional.

Não houve associação estatística significativa ( $p>0,05)$ entre as variáveis "presença de terapia nutricional" e "estado nutricional dos pacientes". Desse modo, propóe-se a realização de novos estudos que abordem o assunto.

\section{Referências}

1. Silva SMCS, Mura JDP. Tratado de alimentação, nutrição e dietoterapia. São Paulo: Roca; 2007.

2. American Diabetes Association. Diagnosis and Classification of Diabetes Mellitus. Diabetes care [Internet]. 2012 [citado em 20 jan. 2012];35(1):64-71. Disponível em: http://care. diabetesjournals.org/content/35/Supplement_1/S64.full. pdf + html3.

3. Isley WL, Molitch ME. Diabetes tipo I. J Clin Endocrinol Metab. 2005;90(1):1.

4. Silva I, Ribeiro JP , Cardoso H. Adesão ao tratamento do diabetes mellitus: a importância das características demográficas e clínicas. Referência. 2006;1(2):33-41.

5. Ortiz MCA, Zanetti ML. Levantamento dos fatores de risco para diabetes mellitus tipo 2 em uma instituição de 
ensino superior. Rev Lat Am Enfermagem [Internet]. 2001 [citado em 22 nov. 2011];9(3):[5 telas]. Disponível em: http://www.scielo.br/scielo.php?script=sci_arttext\&pi$\mathrm{d}=$ S0104-11692001000300009\&lng=pt\&nrm=iso

6. Romero LG, Charro AL, Calle-Pascual AL. Índice glucémico y tratamiento nutricional de las personas con diabetes mellitus. Endocrinol Nutr. 2002;49(7):232-9.

7. Tratamento e Acompanhamento do Diabetes Mellitus. Diretrizes da Sociedade Brasileira de Diabetes Sociedade Brasileira de Diabetes. Princípios para Orientação Nutricional no Diabetes Mellitus. 2006.

8. Oviedo Mota M, Espinoza Larrańaga F, Reyes Morales H, Trejo y Pérez J, Gil Velásquez E. Guía clínica para el diagnóstico y tratamiento de la diabetes mellitus tipo 2 . Rev Med IMSS. 2003;41(1 supl):S27-S46.

9. Boas LCG-V, Foss CF, Foss-Freitas MC, Torres HC, Monteiro LZ, Pace AE. Adesão à dieta e ao exercício físico das pessoas com diabetes mellitus. Texto contexto - enferm. [Internet]. 2011 [citado em 12 fev. 2012];20(2):[7 telas] Disponível em: http:// www.scielo.br/scielo.php?script $=s c i \_a r t t e x t \& p i-$ $\mathrm{d}=$ S0104-07072011000200008\&lng=pt\&nrm=iso

10. Ferreira EAP, Fernandes,AL. Treino em Auto-Observação e Adesão à Dieta em Adulto com Diabetes Tipo 2. Psic: Teor. e Pesq. [Internet]. 2009 [citado em 16 dez. 2011];25(4):[7 telas]. Disponível em: http://www.scielo.br/pdf/ptp/v25n4/ a19v25n4.pdf

11. Barsaglini RA, Canesqui AM . A alimentação e a dieta alimentar no gerenciamento da condição crônica do diabetes. Saude soc. [Internet]. 2010 [citado em 20 ago. 2011];19(4):[13 telas]. Disponível em: http://www.scielo.br/scielo.php?script=sci_arttext\&pi$\mathrm{d}=$ S0104-12902010000400018\&lng=es\&nrm=iso

12. Xavier ATF, Bittar DB, Ataide MBC. Crenças no autocuidado em diabetes: implicaçóes para a prática. Texto contexto - enferm.[Internet]. 2007 [citado em 14 ago. 2011];18(1):[6 telas]. Disponível em: http:// www.scielo.br/scielo.php?script=sci_arttext\&pi$\mathrm{d}=$ S0104-07072009000100015\&lng=es\&nrm=iso

13. Castell GS, Sagnier LB. Larousse da dieta e da Nutrição. São Paulo: Larousse do Brasil; 2004.
14. World Health Organization. Physical status: the use and interpretation of anthropometry. Report of a Who expert committee. Geneva: World Health Organization; 1995.

15. The Nutrition Screening Initiative. Incorporating Nutrition Screening and Interventions into Medical Practice: a monograph for physicians. Washington (DC): The American Dietetic Association; 1994.

16. Cotta RMM, Reis RS, Batista KCS, Dias G, Alfenas RCG, Castro FAF. Hábitos e práticas alimentares de hipertensos e diabéticos: repensando o cuidado a partir da atenção primária. Rev. Nutr. [Internet]. 2009 [citado em 11 jan. 2012];22(6):[12 telas]. Disponível em: http://www.scielo.br/scielo.php?script=sci_arttext\&pi$\mathrm{d}=$ S1415-52732009000600004\&lng $=\mathrm{pt} \& \mathrm{nrm}=$ iso

17. Gomes MB, Neto DG, Mendonça E, Tambascia MA, Fonseca RM, Réa RR, et al. Prevalência de Sobrepeso e Obesidade em Pacientes Com Diabetes Mellitus do Tipo 2 no Brasil: Estudo Multicêntrico Nacional. Arq. Bras. Endocrinol. Metab. 2006;50(1):136-44.

18. The ACCORD Study Group. Effects of Combination Lipid Therapy in Type 2 Diabetes Mellitus. N Engl J Med [Internet]. 2010 [citado em 12 jan 2012];362(17):1563-74. Disponível em: http://europepmc.org/articles/PMC2879499/ reload $=0$; jsessionid=UiU4wUtMZNdsLfrXUKBq.6

19. Pontieri FM, Bachion MM. Crenças de pacientes diabéticos acerca da terapia nutricional e sua influência na adesão ao tratamento. Cien Saude Colet. 2010;15(1):151-60.

20. Cuppari L. Guia de Nutrição: nutrição clínica no adulto. São Paulo: Manole, 2005.

21. Costa ACP, Thalacker M, Besenbruch N, Simony RF, Branco, FC. Metabolic and nutritional aspects of carbohydrate counting in the treatment of type 1 diabetes mellitus. Nutrire: rev. Soc. Bras. Alim. Nutr. = J. Brazilian Soc. Food Nutr. 2011;36(1):151-62.

22. Costa JA, Balga RSM, Alfenas RCG Cotta, RMM. Promoçáo da saúde e diabetes: discutindo a adesão e a motivação de indivíduos diabéticos participantes de programas de saúde. Cien Saude Colet. 2011;16(3):2001-9.23.

23. Fontinelle RSS, Peres LCL, Nascimento MAB, Boni MS. Avaliação do conhecimento sobre alimentação entre pacientes com diabetes tipo 2. Com Cien Saude. 2007;18(3):197-206. 